\title{
Serum Prolactin Levels in Patients with Major Depressive Disorder Receiving Selective Serotonin-Reuptake Inhibitor Monotherapy for 3 Months: A Prospective Study
}

\author{
Young-Min Park ${ }^{\bowtie}$ \\ Department of Psychiatry, Ilsan Paik Hospital, Inje University College of Medicine, Goyang, Republic of Korea
}

Objective It is unclear whether selective serotonin-reuptake inhibitors (SSRIs) can significantly increase the prolactin level. The purpose of this study was to identify the relationship between the prolactin level and the administration of SSRIs such as escitalopram and sertraline. An additional purpose was to determine whether the elevation of prolactin differs between escitalopram and sertraline treatment.

Methods Serum prolactin levels were measured at baseline and after 3 months in 23 patients who received SSRI monotherapy with escitalopram ( $\mathrm{n}=18$ ) (ESC group) or sertraline $(\mathrm{n}=5)$ (SERT group) for 3 months.

Results The prevalence of hyperprolactinemia at posttreatment was $34.8 \%(8 / 23)$. The overall pretreatment and posttreatment prolactin levels were $21.86 \pm 20.21$ and $19.89 \pm 12.03 \mathrm{ng} / \mathrm{mL}$ (mean $\pm \mathrm{SD}$ ), respectively, with ranges of $6.85-86.20 \mathrm{and} 5.19-47.61 \mathrm{ng} / \mathrm{mL}$. The pretreatment and posttreatment prolactin levels were $20.66 \pm 15.92$ and $21.97 \pm 12.33 \mathrm{ng} / \mathrm{mL}$, respectively, in the ESC group, and $26.18 \pm 33.75$ and $12.43 \pm 7.76 \mathrm{ng} / \mathrm{mL}$ in the SERT group.

Conclusion Clinicians should be aware that hyperprolactinemia can appear in patients receiving escitalopram or sertraline, even though they do not need routine monitoring for prolactin levels.

Psychiatry Investig 2017;14(3):368-371

Key Words SSRI, Serotonin, Prolactin, Hyperprolactinemia.

\section{INTRODUCTION}

It is some evidence that antidepressants that inhibit serotonin reuptake can increase the prolactin level. This adverse effect has been called serotonin overactivity. Selective serotonin-reuptake inhibitors (SSRIs) have been reported as the most common cause of drug-induced hyperprolactinemia. ${ }^{1} \mathrm{~A}$ pharmacoepidemiological study using a pharmacovigilance database found that SSRIs were clearly associated with an increased risk of hyperprolactinemia. ${ }^{2,3}$ The mechanisms of SS$\mathrm{RI}$-induced hyperprolactinemia is not clear. Some investigators suggested this hyperprolactinemia is associated with the serotonin indirect effect via serotonin receptors and the sero-

Received: April 16, 2016 Revised: July 22, 2016

Accepted: September 21, 2016 Available online: January 22, 2017

$\triangle$ Correspondence: Young-Min Park, MD, PhD

Department of Psychiatry, Ilsan Paik Hospital, Inje University College of Medicine, 170 Juhwa-ro, Ilsansu-gu, Goyang 10380, Republic of Korea Tel: +82-31-910-7260, Fax: +82-31-910-7268, E-mail: medipark@hanmail.net

(a) This is an Open Access article distributed under the terms of the Creative Commons Attribution Non-Commercial License (http://creativecommons.org/licenses/bync/4.0) which permits unrestricted non-commercial use, distribution, and reproduction in any medium, provided the original work is properly cited.
tonin-GABA-dopamine interaction. ${ }^{4}$

However, some studies have found that SSRIs do not significantly increase prolactin levels relative to baseline. ${ }^{4}$ Some investigators have reported that sertraline $\mathrm{e}^{5}$ and citalopram ${ }^{6} \mathrm{did}$ not increase prolactin levels significantly. It is therefore unclear whether SSRIs increase prolactin levels significantly. Moreover, there have been few studies involving escitalopram and prolactin.

The purpose of this study was to identify the relationship between prolactin level and the administration of SSRIs such as escitalopram and sertraline. An additional purpose was to determine whether the elevation of prolactin differs between escitalopram and sertraline treatment.

\section{METHODS}

\section{Subjects}

This study involved 23 outpatients who met the criteria of the Fourth Edition of the Diagnostic and Statistical Manual of Mental Disorders for major depressive disorder (MDD). Subjects in whom the prolactin levels were measured at baseline 
and after 3 months were analyzed. They had no history of antidepressant treatment within the 3 months immediately prior to first visiting our hospital. The only comedication allowed was benzodiazepine. In addition, the subjects had no medical condition affecting prolactin levels, such as pituitary disorders, CNS disorders, or systemic diseases. Written informed consent to participate was obtained from all patients before beginning the investigation. The study and its protocol were approved by our institutional review board.

\section{Study design}

Serum prolactin levels were measured at two time points (baseline and 3 months) in 23 patients who received SSRI monotherapy with escitalopram $(n=18)$ (ESC group) or sertraline ( $\mathrm{n}=5$ ) (SERT group) for 3 months. A blood sample was taken in the morning and then centrifuged to separate serum. The upper normal range of the prolactin level was considered to be $20 \mathrm{ng} / \mathrm{mL}$ in men and $25 \mathrm{ng} / \mathrm{mL}$ in women, with hyperprolactinemia defined as being present when the prolactin level was higher than these limits.

\section{Measurements of prolactin}

The concentration of prolactin was measured using a screening assay kit (Human Magnetic Luminex, R\&D Systems, Minneapolis, MN, USA). A microparticle cocktail was injected into each microplate of the screening assay kit, and then incubation and washing were performed. A biotin antibody cocktail was then injected, followed again by incubation and washing. Finally, streptavidin-phycoerythrin was injected, and the level of prolactin was measured after further incubation and washing.

\section{Analysis}

Variables such as demographic or clinical values, prolactin levels, and the frequencies of group parameters were compared using the Mann-Whitney test, chi-square test, and Spearman's correlation analysis as appropriate. All tests were twotailed, and group differences were tested at the $\mathrm{p}<0.05$ level. All statistical analyses were carried out using SAS (version 9.3, SAS Institute, Cary, NC, USA) or SALT (version 2.5, ISTECH Corporation, Goyang, Korea).

\section{RESULTS}

The prevalence rates of hyperprolactinemia at pretreatment and posttreatment were $21.7 \%(6 / 23)$ and $34.8 \%(8 / 23)$, respectively. However, the prolactin levels in two hyperprolactinemic patients at pretreatment decreased to normal levels at posttreatment (one each in the ESC and SERT groups), while those of four normoprolactinemic patients at pretreatment became hy- perprolactinemic at posttreatment (three in the ESC group and one in the SERT group). The mean prolactin levels in pretreatment and posttreatment between male and female subjects did not differ significantly $(18.53 \pm 4.01 \mathrm{ng} / \mathrm{mL}$ vs. $22.56 \pm 22.22 \mathrm{ng} / \mathrm{mL}$; $20.34 \pm 5.25 \mathrm{ng} / \mathrm{mL}$ vs. $19.81 \pm 13.12 \mathrm{ng} / \mathrm{mL}$ ).

The overall pretreatment and posttreatment prolactin levels were $21.86 \pm 20.21$ and $19.89 \pm 12.03 \mathrm{ng} / \mathrm{mL}$, respectively, with ranges of $6.85-86.20$ and 5.19-47.61 ng/mL (Table 1). The pretreatment and posttreatment prolactin levels were $20.66 \pm 15.92$ and $21.97 \pm 12.33 \mathrm{ng} / \mathrm{mL}$, respectively, in the ESC group, and $26.18 \pm 33.75$ and $12.43 \pm 7.76 \mathrm{ng} / \mathrm{mL}$ in the SERT group. The mean escitalopram and sertraline dosages were 10.3 and 70 mg, respectively.

The mean prolactin levels did not differ significantly between pretreatment and posttreatment among the 23 patients ( $>0.05$ ) (Figure 1). In addition, the mean prolactin levels at both posttreatment and pretreatment did not differ significantly between the ESC and SERT groups, although there was

Table 1. Comparison of pretreatment clinical variables

\begin{tabular}{lc}
\hline \multicolumn{1}{c}{ Variable } & Value $(\mathrm{N}=23)$ \\
\hline Age (years) & $42.13 \pm 14.28$ \\
Sex (male/female) & $4 / 19$ \\
Pretreatment BDI score & $27.35 \pm 9.26$ \\
Pretreatment HAMD score & $16.57 \pm 5.15$ \\
Posttreatment BDI score & $13.28 \pm 11.62$ \\
Posttreatment HAMD score & $7.13 \pm 4.64$ \\
Pretreatment prolactin $(\mathrm{ng} / \mathrm{mL})$ & $21.86 \pm 15.55$ \\
Posttreatment prolactin $(\mathrm{ng} / \mathrm{mL})$ & $19.90 \pm 16.24$ \\
\hline
\end{tabular}

Except where indicated otherwise, the data are mean \pm SD or N/N values. BDI: Beck Depression Inventory, HAMD: Hamilton Depression Rating Scale

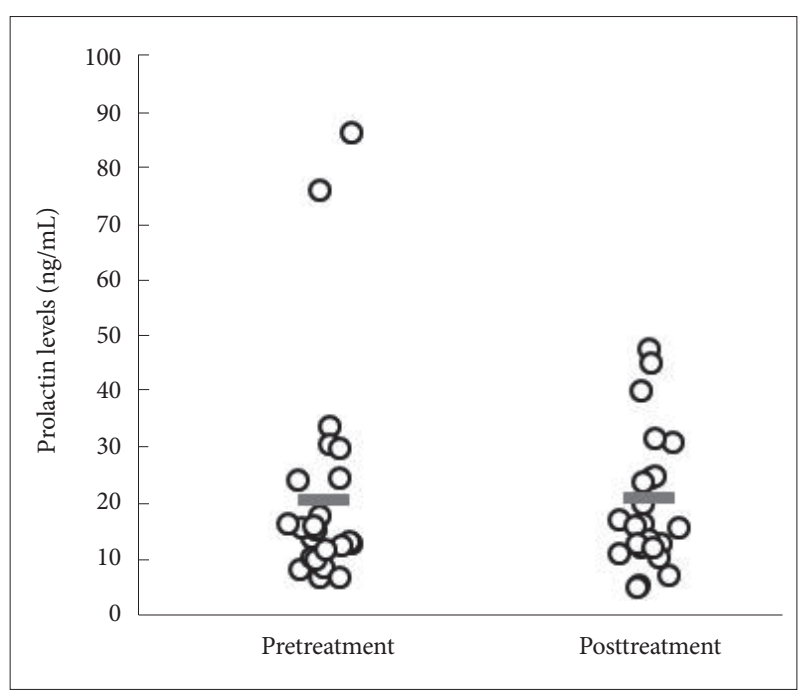

Figure 1. Comparison of the mean prolactin level (gray bars) between pretreatment and posttreatment. 
a tendency for the levels to be lower in the SERT group than in the ESC group at posttreatment $(\mathrm{p}=0.074)$ (Supplementary Figures 1 and 2 in the online-only Data Supplement).

\section{DISCUSSION}

This study found that the prevalence of hyperprolactinemia induced by administering escitalopram and sertraline for 3 months was $34.8 \%$. This rate is higher than that found after administering fluoxetine for 3 months by previous investigators $(12.5 \%)^{7}$ and after administering SSRIs for more than 1 year in our previous cross-sectional study $(10.9 \%) .{ }^{8}$ However, six patients in the present study already had hyperprolactinemia at the pretreatment stage. After SSRI treatment, the prolactin levels in two of these six patients had normalized, while four patients were still hyperprolactinemic. In addition, four patients had new-onset hyperprolactinemia. Allowing for this, the prevalence of new-onset SSRI-induced hyperprolactinemia was $17.4 \%(4 / 23)$.

It was unclear whether hyperprolactinemia was present in some of the patients at pretreatment. It has been hypothesized that a stressful state is associated with hyperprolactinemia. ${ }^{9}$ This is supported by the prolactin levels of two patients with hyperprolactinemia at pretreatment decreasing to almost normal or normal levels after SSRI treatment in the present study (from 75.9 to $30.8 \mathrm{ng} / \mathrm{mL}$ and from 86.2 to $12.1 \mathrm{ng} / \mathrm{mL}$ ). The mechanism by which SSRIs affect prolactin secretion is also unclear. There are two hypothesis. First, serotonin modulates prolactin secretion indirectly via postsynaptic $5 \mathrm{HT} 1 \mathrm{~A}$, 5HT1C/2, 5-HT2, 5-HT2C, and 5HT3 receptor. ${ }^{10}$ Second, serotonin inhibits GABA interneuron and this leads to weaken the inhibition of dopaminergic action over prolactin. At last it causes increased prolactin secretion. ${ }^{10}$

The prolactin levels at pretreatment did not differ between the ESC and SERT groups, whereas after 3 months of treatment there was a nonsignificant tendency for the level to be lower in the SERT group than in the ESC group $(\mathrm{p}=0.074)$. This can be explained by sertraline being applied at a very low dosage (mean $70 \mathrm{mg}$ ) and it having the unique pharmacological property of potent dopamine-reuptake inhibition. ${ }^{11}$ It is also possible that this dopaminergic action at least partially counteracted its simulation of serotonin activity or serotoninreuptake inhibition at a low dosage. Some investigators also found that sertraline did not affect the prolactin levels, although the dosage was low in those studies. ${ }^{5,12}$ However, another study found the opposite result, with the prevalence of SSRI-induced hyperprolactinemia being highest when administering sertraline. ${ }^{2}$ Similarly, there are also conflicting results for escitalopram from the small number of relevant studies. ${ }^{3,13}$ The present study found that sertraline and escitalopram did not induce dramatic changes in the prolactin levels. The highest prolactin levels were 47.6 and $23.47 \mathrm{ng} / \mathrm{mL}$ in the ESC and SERT groups, respectively, implying that SSRIs do not induce dramatic changes in prolactin, which contrasts with the known effects of some kinds of antipsychotics.

This study was subject to several limitations. The sample was very small, especially in the sertraline group, and so studies involving larger samples are needed in the future. Also, the mean dosages of escitalopram and sertraline were low, and it is possible that different results would be obtained when using higher dosages. In addition, the normal prolactin range when using the Human Magnetic Luminex screening assay kit was not established clearly. In spite of these limitations, this study supports that SSRIs can induce hyperprolactinemia in some patients with MDD. Clinicians should therefore be aware that this adverse effect can appear in patients receiving escitalopram or sertraline, even though they do not need routine monitoring for prolactin levels.

\section{Supplementary Materials}

The online-only Data Supplement is available with this article at https://doi.org/10.4306/pi.2017.14.3.368.

\section{Acknowledgments}

This study was supported by a grant from the National Research Foundation of Korea (NRF), funded by the Ministry of Education (grant no. NRF2014R1A1A2A10059345).

\section{REFERENCES}

1. Torre DL, Falorni A. Pharmacological causes of hyperprolactinemia. Ther Clin Risk Manag 2007;3:929-951.

2. Petit A, Piednoir D, Germain ML, Trenque T. Drug-induced hyperprolactinemia: a case-non-case study from the national pharmacovigilance database. Therapie 2003;58:159-163.

3. Trenque T, Herlem E, Auriche P, Drame M. Serotonin reuptake inhibitors and hyperprolactinaemia: a case/non-case study in the French pharmacovigilance database. Drug Saf 2011;34:1161-1166.

4. Coker F, Taylor D. Antidepressant-induced hyperprolactinaemia: incidence, mechanisms and management. CNS Drugs 2010;24:563-574.

5. Sagud M, Pivac N, Muck-Seler D, Jakovljevic M, Mihaljevic-Peles A, Korsic M. Effects of sertraline treatment on plasma cortisol, prolactin and thyroid hormones in female depressed patients. Neuropsychobiology 2002;45:139-143.

6. Hawken ER, Owen JA, Van Vugt D, Delva NJ. Effects of oral racemic citalopram on neuroendocrine responses. Prog Neuropsychopharmacol Biol Psychiatry 2006;30:694-700.

7. Papakostas GI, Miller KK, Petersen T, Sklarsky KG, Hilliker SE, Klibanski A, et al. Serum prolactin levels among outpatients with major depressive disorder during the acute phase of treatment with fluoxetine. J Clin Psychiatry 2006;67:952-957.

8. Kim S, Park YM. Serum prolactin and macroprolactin levels among outpatients with major depressive disorder following the administration of selective serotonin-reuptake inhibitors: a cross-sectional pilot study. PLoS One 2013;8:e82749.

9. Spiers JG, Chen HJ, Bradley AJ, Anderson ST, Sernia C, Lavidis NA. Acute restraint stress induces rapid and prolonged changes in erythrocyte and hippocampal redox status. Psychoneuroendocrinology 2013; 38:2511-2519. 
10. Emiliano AB, Fudge JL. From galactorrhea to osteopenia: rethinking serotonin-prolactin interactions. Neuropsychopharmacology 2004;29: 833-846.

11. Richelson E. Interactions of antidepressants with neurotransmitter transporters and receptors and their clinical relevance. J Clin Psychiatry 2003;64(Suppl 13):5-12.
12. Gordon C, Whale R, Cowen PJ. Sertraline treatment does not increase plasma prolactin levels in healthy subjects. Psychopharmacology (Berl) 1998;137:201-202.

13. Nadeem HS, Attenburrow MJ, Cowen PJ. Comparison of the effects of citalopram and escitalopram on 5-Ht-mediated neuroendocrine responses. Neuropsychopharmacology 2004;29:1699-1703. 